\title{
CULTURA Y SOCIEDAD
}

\author{
Análisis de la relación entre \\ nuevas tecnologías y chino \\ como segunda lengua: aplicaciones \\ para teléfonos inteligentes y juegos
}

\section{Analysis of the Relationship between New Technologies and Chinese as a Second Language: Mobile Phone Applications and Games}

\author{
GABRIEL TEROL ROJO* \\ TAO LI*
}

\section{Introducción}

Se ofrece un estudio sistemático y el análisis de la documentación más destacada y actual sobre la relación entre aprendizaje y enseñanza de una segunda lengua (L2), aplicada a la lengua china (CSL, chino como segunda lengua). Luego de repasar la trayectoria de los avances tecnológicos, en particular de las aplicaciones (apps) de telefonía celular y el diseño u oferta de juegos y simuladores para la enseñanza y el aprendizaje de CSL, se hace referencia a artículos e investigaciones especializados publicados en revistas internacionales, tanto sobre la lengua china como de lingüística y filología comparadas. Con ello

Recepción: 23 de agosto de 2016. / Aceptación: 13 de octubre de 2016.

* Universidad de Valencia, Gabriel.Terol@uv.es / Shanghai International Studies University, litao@shisu.edu.cn 
se actualiza el listado de trabajos pertinentes, entre los que se encuentran tesis de maestría chinas en la materia, actas de congresos internacionales de enseñanza de la lengua china y artículos de revistas de facultades de educación sobre la modernización de la enseñanza de la lengua china o del CSL; también se presentan datos de consumo y preferencias de usuarios que evalúan las opciones que el mercado tecnológico (atendiendo a los diferentes sistemas operativos) ofrece. Los trabajos derivados de la investigación realizada por la Sociedad Internacional para la Enseñanza del Idioma Chino (世界汉语教学学会) y la colaboración de la Oficina del Consejo Internacional de la Lengua China o Hanban (国家汉办) y su organización diseminada por todo el mundo como Instituto Confucio (孔子学院), confirman y validan la importancia de los recursos tecnológicos en la enseñanza de CSL.

Los resultados que se destacan son producto del análisis bibliográfico de publicaciones científicas internacionales especializadas en la aplicación de la tecnología a la enseñanza de lenguas (como al CSL), así como de programas y manuales de reciente edición. Tanto las conclusiones de estos trabajos como la reflexión que suscitan brindan una simplificación metodológica de la circunstancia actual. Con ellas se destacan las fortalezas y las debilidades principales de las más recientes y relevantes aplicaciones para telefonía celular, los juegos en red y los simuladores de realidad virtual.

\section{Análisis y resultados}

La actualización sobre la aplicación de las tecnologías a la enseñanza de CSL ayuda a comprender el camino que lleva esta relación. El trabajo de Qianqian Xiong ${ }^{1}$ recoge los recursos bibliográficos publicados a raíz de reuniones como la Conferencia General Internacional del Instituto Confucio (全球孔子学院 大会), el Congreso Internacional de Información y Tecnología

${ }^{1}$ Qianqian Xiong, “近十年来现代教育技术在汉语国际教育中的应用及趋势” [Aplicación y tendencia de las tecnologías modernas en la enseñanza internacional de chino en la última década], tesis de maestría, Universidad de Estudios Extranjeros de Guangdong, 2014, p. 18. 
Educativas (国际教育信息技术大会), el Conferencia Internacional de Enseñanza de Chino (国际汉语教学大会), el Congreso Internacional de Educación de Chino como Segunda Lengua (国际对外汉语教学大会) y el Congreso Internacional de Enseñanza-Aprendizaje por Internet y Juegos (国际网络教学及游戏 大会), y resume los porcentajes de uso de distintas tecnologías en la enseñanza del chino (cuadro 1).

\section{Cuadro 1. Porcentaje de uso tecnológico}

\begin{tabular}{lc}
\hline Nuevas tecnologías & Porcentaje \\
\hline Tecnologías 3G, teléfonos inteligentes, juegos, etc. & 47 \\
Tecnologías multimedia & 23 \\
Online & 13 \\
A distancia & 5 \\
Sistemas de telecomunicación & 6 \\
Software educativos & 3 \\
Videos y programas de TV & 3 \\
\hline
\end{tabular}

El alto porcentaje de usuarios de plataformas educativas para aprender chino a través de tecnologías, teléfonos inteligentes, juegos y simuladores, permite valorar su aportación a la enseñanza y el aprendizaje de CSL.

\section{Aplicaciones para teléfonos inteligentes}

Según las estadísticas de las páginas web más grandes de descarga de software para teléfonos inteligentes en China (básicamente Sky Software, Pacific Online y Xiazai Ba), las aplicaciones más populares, de acuerdo con Xiong, son: ${ }^{2}$ 悟空识字 (Aprender caracteres chinos con Wukong), 熊猫识字 (Aprender caracteres chinos Panda), 6小时练字 (Aprender a escribir chino en seis horas), 新华字典 (Diccionario Xinhua) y Super Speech para el aprendizaje de la pronunciación. Asimismo, es muy conocida 
la aplicación Chinese Walking (中文漫步), desarrollada por organizaciones francesas del Instituto Confucio de la Universidad de Poitiers y el Centro Nacional de Educación a Distancia.

Un estudio de Zhizhi Liang ${ }^{3}$ sobre el diseño de software de teléfono inteligente para el aprendizaje de vocabulario de chino para estudiantes extranjeros clasifica las aplicaciones actuales para el sistema Android. La primera clasificación da cuenta del contenido de estas aplicaciones (cuadro 2); otra las ordena en función del tipo de software (cuadro 3); la siguiente destaca los idiomas de estas aplicaciones (cuadro 4) y, por último, las organiza teniendo en cuenta la cantidad de descargas (cuadro 5).

CuAdro 2. Contenido de las aplicaciones

\begin{tabular}{lrc}
\hline Contenido & Cantidad & Porcentaje \\
\hline Aprendizaje de destrezas integrales & 33 & 48.5 \\
Conversación 3, Audiovisual 2, Lectura 1 & 6 & 12.5 \\
Fonética 5, Vocabulario 7, Gramática 1 & 13 & 19.1 \\
Aprendizaje para HSK & 12 & 17.6 \\
Herramienta & 4 & 5.9 \\
\hline
\end{tabular}

CuAdro 3. Tipo de software en aplicaciones

\begin{tabular}{lcc}
\hline Tipo de software & Cantidad & Porcentaje \\
\hline Software flashcard & 7 & 10.3 \\
Software de juegos & 6 & 8.8 \\
Software de aprendizaje & 55 & 80.9 \\
\hline Total & 68 & 100 \\
\hline
\end{tabular}

Los resultados pertinentes muestran con gran claridad que el software relacionado con el aprendizaje es el más descargado

${ }^{3}$ Zhizhi Liang, “面向初级汉语水平外国学生词汇学习的手机汉语学习软件 设计” [Diseño de software de teléfono inteligente para el aprendizaje de vocabulario chino para estudiantes extranjeros de nivel inicial], 广西教育学院学报 [Revista del Colegio de Educación de Guangxi], vol. 3, 2014, pp. 152-155. 
(80.9\%); en cuanto al contenido, el que apuesta por destrezas integrales es el más presente (48.5\%); el inglés es la lengua más utilizada (91.1\%), y de las 68 aplicaciones, dos de ellas cuentan con gran popularidad (10000 descargas), mientras que $67 \%$ tienen una descarga notablemente menor (entre 100 y 500).

CuAdro 4. Idioma de las aplicaciones

\begin{tabular}{lcc}
\hline Idioma & Cantidad & Porcentaje \\
\hline Inglés & 62 & 91.1 \\
Japonés & 4 & 5.9 \\
Ruso & 1 & 1.5 \\
Francés & 1 & 1.5 \\
\hline Total & 68 & 100 \\
\hline
\end{tabular}

Cuadro 5. Cantidad de descargas

\begin{tabular}{lcc}
\hline Número de descargas & Cantidad & Porcentaje \\
\hline 10000 & 2 & 2.9 \\
$5000-10000$ & 2 & 2.9 \\
$1000-5000$ & 8 & 11.8 \\
$500-1000$ & 10 & 14.7 \\
$100-500$ & 46 & 67.7 \\
\hline
\end{tabular}

En cuanto al entorno geográfico de China, podemos atender a tres estudios que dan cuenta del interés que existe en aplicar las tecnologías a la enseñanza de CSL en Bangladesh, Corea del Sur y Hong Kong. Estos trabajos dan cuenta de la expansión de plataformas y la comercialización de programas que utilizan el teléfono inteligente como instrumento educativo tanto en China continental como en su entorno geográfico sirviéndose de herramientas comunes.

En ese sentido, las más usadas para la enseñanza y el aprendizaje de CsL del software social más popular, WeChat (Weixin), 
son las siguientes: 元任对外汉语 (Aprender chino), 对外汉语 人俱乐部 (Club para aprender chino) y 对外汉语网 (Enseñar chino como lengua extranjera), así como 北语RICH对外汉 语 (programa RICH de la Universidad de Lengua y Cultura de Beijing). Meng Gang Xiao, ${ }^{4}$ a partir del análisis del uso de estas aplicaciones para teléfonos inteligentes en el aprendizaje del chino por parte de un centenar de alumnos universitarios de Bangladesh, concluye, sobre las fortalezas y las debilidades de estos sistemas, que entre las primeras destaca la complementariedad de estos materiales respecto a la enseñanza en el aula y su innegable libertad de uso; puede recurrirse a ellos en diferentes momentos y circunstancias. Entre sus debilidades señala discrepancias en los contenidos gramaticales de diversas aplicaciones que aún no son lo suficientemente sencillos; en las de fonética, la calidad del audio no siempre es la deseable; en las aplicaciones para comprobar el nivel de competencia en lengua china (Hànyǔ Shuǐpíng Kăoshì, HSK) faltan prácticas, posiblemente porque requieren más tiempo y una tecnología más avanzada; finalmente, reconoce la escasez de aplicaciones profesionales de gramática china.

El trabajo de Youmi Moon ${ }^{5}$ analiza las aplicaciones disponibles en Corea en los sistemas Android e iOS y los clasifica en función de sus contenidos y temáticas (cuadros 6 y 7).

Sus resultados muestran cómo en ambos sistemas operativos hay una clara apuesta por contenidos similares: expresión oral, vocabulario y diccionarios $(38,19$ y $11 \%$ para Android, y 25,23 y $8 \%$ para iOS). Los contenidos relacionados con la prueba oficial de nivel HSK compartirían, más o menos, relevancia (6 y $4 \%$ respectivamente). Destaca, en el ámbito global, la importancia que tienen para iOS los contenidos relacionados con la lectura y la cultura (13 y 12\%) en comparación con

${ }^{4}$ Meng Gang Xiao, “汉语学习类手机应用使用情况调查研究一以孟加拉达卡 大学汉语学习者为例” [Investigación del uso de aplicaciones de teléfono inteligente de enseñanza de chino. Análisis basado en alumnos de chino de la Universidad de Dhaka de Bangladesh], tesis de maestría, Universidad de Yunnan, 2015.

5 Youmi Moon, “基于智能手机app的汉语口语智能学习模式设计研究一以韩 国初级汉语学习者为对象” [Estudio del uso de las aplicaciones para el aprendizaje inteligente: el caso de los estudiantes coreanos y su aprendizaje aplicado a conversación], 中文教学现代化学报 [Revista para la Modernización de la Enseñanza de la Lengua China], vol. 4, núm. 2, 2015. 
Cuadro 6. Contenidos y temáticas en Android

\begin{tabular}{lr}
\hline Temáticas & Porcentaje \\
\hline Diccionarios & 11 \\
HSK & 6 \\
Vocabulario & 19 \\
Cultura & 2 \\
Lectura & 8 \\
Expresión oral & 38 \\
\hline
\end{tabular}

\section{Cuadro 7. Contenidos y temáticas en iOS}

\begin{tabular}{lc}
\hline Temáticas & Porcentaje \\
\hline Diccionarios & 8 \\
HSK & 4 \\
Vocabulario & 23 \\
Cultura & 12 \\
Escritura & 8 \\
Lectura & 13 \\
Expresión oral & 25 \\
\hline
\end{tabular}

la que le da Android (8 y 2\%), y en el campo de la escritura el $8 \%$ que le dedica iOS es inexistente en Android.

De modo similar, un trabajo de Liyu Chen y Yongyu Tan ${ }^{6}$ presenta un análisis de los recursos digitales desde un punto de vista fonético de la enseñanza y el aprendizaje de CSL para los hablantes de chino cantonés. Debido a la incipiente relación económica de la población de Hong Kong con la provincia de Guangdong y la China continental y a las notables diferencias entre aquel dialecto y la lengua estándar (putonghua), surgieron propuestas de asistencia tecnológica para la enseñanza fonética

${ }^{6}$ Liyu Chen y Yongyu Tan, “数位资源辅助华语语音教学分析一以香港粤语 母语者为教学对象” [Análisis de recursos digitales de enseñanza y aprendizaje de la fonética en chino mandarín para los hablantes de chino cantonés], 第十二屆台灣華 語文教學年會暨國際學術研討會 [Acta del XII Congreso Internacional de Enseñanza y Aprendizaje de Chino de Taiwan], 屆台: 文藻外語學院應用華語文系暨華語文教學研 究所 [Taiwan, Facultad de Lenguas Extranjeras Aplicadas Wenzao Instituto Superior de Enseñanza de Lengua China], 2013, pp. 351-361. 
de la lengua china a esta población. La clasificación que presenta este estudio destaca las mejores para dicha finalidad (cuadro 8).

CuAdro 8. Aplicaciones de teléfono inteligente para el aprendizaje fonético del chino moderno

\begin{tabular}{|c|c|c|c|}
\hline Nombre & Año & Operador & Enlace \\
\hline $\begin{array}{l}\text { E-shén bāo dōngguā } \\
\text { (E神保冬瓜) }\end{array}$ & 2011 & Noah Media & $\begin{array}{l}\text { http://similarapps.me/E\% } \\
\text { E7\%A5\%9E\%E7\%85\%B2 } \\
\% \text { \%5\%86\%AC\%E7\%93\%9 } \\
\text { CLite/1228255 }\end{array}$ \\
\hline $\begin{array}{l}\text { Pǔtōnghuà liànxí shì } \\
\text { (普通话练习室) }\end{array}$ & 2013 & Fatho & $\begin{array}{l}\text { https://www.appannie. } \\
\text { com/en/apps/ios/app/ } \\
\text { pu-tong-hua-lian-xi-shi/ }\end{array}$ \\
\hline $\begin{array}{c}\text { Fāshēng pǔtōnghuà } \\
\text { biāozhǔn jīchǔ yīnjié } \\
\text { xuéxíi jī } \\
\text { (发声普通话标准基 } \\
\text { 础音节学习机) }\end{array}$ & 2013 & Ace App & $\begin{array}{l}\text { http://appdp.com/app/ } \\
2872 /\end{array}$ \\
\hline $\begin{array}{l}\text { Yinnshíyè shíyòng } \\
\text { pǔtōnghuà huihuà } \\
\text { zìxué kèchéng } \\
\text { (饮食业实用普通话 } \\
\text { 会话自学课程) }\end{array}$ & 2013 & QTSA & $\begin{array}{l}\text { https://play.google.com/ } \\
\text { store/apps/details?id=com. } \\
\text { crossover.qtsa.pth }\end{array}$ \\
\hline $\begin{array}{l}\text { Língshòu yè shíyòng } \\
\text { pưtōnghuà huihuà } \\
\text { zìxué kèchéng } \\
\text { (零售业实用普通话 } \\
\text { 会话自学课程) }\end{array}$ & 2013 & QTSA & $\begin{array}{l}\text { https://itunes.apple.com/ } \\
\text { cn/app/\%E9\%9B\%B6\%E5 } \\
\text { \%94\%AE\%E4\%B8\%9A\%E } \\
\text { 5\%AE\%9E\%E7\%94\%A8\% } \\
\text { E6\%99\%AE\%E9\%80\%9A } \\
\text { \%E8\%AF\%9D\%E4\%BC\% } \\
\text { 9A\%E8\%AF\%9D\%E8\%87 } \\
\text { \%AA\%E5\%AD\%A6\%E8\% } \\
\text { AF\%BE\%E7\%A8\%8B-lite/ } \\
\text { id561905303?mt=8 }\end{array}$ \\
\hline
\end{tabular}

Entre los resultados destacan aplicaciones sobre actividades prácticas específicas. Por un lado está la relacionada con el sec- 
tor agrícola y de la alimentación, y por otro, desde un punto de vista más genérico, la vinculada a transacciones comerciales (compra y venta). Sin duda, sirven de referencia para satisfacer las principales necesidades lingüísticas, pero no resultarán excluyentes ni exclusivas en un futuro inmediato.

\section{Juegos y simuladores}

Una visión panorámica de la situación actual exige mencionar las principales ofertas en $3 \mathrm{D}$ relacionadas con el tema. El mundo virtual en línea de ActiveWorlds, creado en Massachusetts en 1995, es un buen ejemplo. Al ofrecer a los usuarios la posibilidad de explorar mundos y entornos virtuales, y la creación personalizada de contenidos propios, su aplicación a la enseñanza de idiomas y del chino en particular es muy popular. Twinity, por su parte, comenzó a funcionar en 2008, y promete navegar virtualmente por versiones históricas de las ciudades del mundo. HIPIHI, activo de 2007 a 2012, es la versión china de estos simuladores de realidad virtual que tantas ventajas ofrecen para la enseñanza del chino. Esta revisión podría cerrarse con Open Cobalt, de 2009, y Opensim, de 2014.

Entre la oferta actual en China para aprender CSL, y siguiendo la tesis de Xiong, destacan simuladores de procedencia coreana. Todos se centran en la enseñanza de caracteres chinos y ya tienen cierto tiempo circulando. En orden cronológico, destaca el videojuego de RPG (Role-Playing Game), desarrollado por la empresa NHN Entertainment en 2009, 1lamado Hanjamaru (汉字房), así como el videojuego de la compañía NCSOFT en 2010, Magic Hanja (魔法千字文OL). Ambos apoyan sus objetivos pedagógicos de escritura y reconocimiento de caracteres en juegos desarrollados en red y basados en la moderna teoría del EFM (Effective Learning Environment, Flow, Motivation).?

El trabajo de Xiao, por su parte, proporciona otras referencias. La primera la desarrolla la Oficina General del Instituto

7 Yi Zhang et al., "Educational Game Design for Teaching Chinese as a Foreign Language by Effective Learning Environment, Flow, Motivation", en Xiangfeng Luo et al. (eds.), New Horizons in Web-based Learning-ICWL 2010 Workshops, BerlínHeidelberg, Springer, 2011. 
Confucio (organismo oficial encargado de la enseñanza de la lengua china fuera de China) en colaboración con la Universidad Estatal de Michigan. Se trata de Zon: The New Chengo Chinese (新乘风汉语), un juego virtual en red. Creado en 2009, promueve un entorno sugerente y propicia el EFM mediante una progresión de contenido (hasta cinco niveles). Su interactividad aumenta su atractivo entre los usuarios y posibilita un aprendizaje sin esfuerzo. La segunda opción la ofrece el Laboratorio Linden de Estados Unidos: Second Life (第二人 生). Activo desde 2003 como juego en red, en 2007 empezó a utilizarse como herramienta en la enseñanza de idiomas. En la actualidad cuenta con más de ocho millones de usuarios. Sin duda, ha desempeñado un papel tan importante en la enseñanza de CSL que merece ser considerado. Los casos que documenta la investigación de Shijuan $\mathrm{Liu}^{8}$ así lo demuestran. Ambas iniciativas son accesibles en el mercado americano, al alcance de los interesados en aprender chino.

La creación de espacios virtuales en la plataforma por Second Life generó buenos resultados de aprendizaje, como lo especifican los trabajos realizados por Scott Grant, del Programa de Estudios Chinos de la Universidad de Monash en Melbourne, Australia; Sachiko Kamioka, del Centro de Estudios de Segundas Lenguas Aplicadas de la Universidad de Oregon; Zheng Xiuren, de la Universidad Cristiana Chung Yuan de Taiwan; Zhan Hong, de la Universidad Aeronáutica EmbryRiddle de Arizona, y Chen Qingxuan, de la Academia China de Ciencias. ${ }^{9}$ Todos son ejemplos del interés que este programa ha suscitado en la enseñanza de CSL. La investigación doctoral

${ }^{8}$ Shijuan Liu, “Second Life” 及其在中文教学中的应用 [Second Life y su aplicación en la enseñanza y en el aprendizaje del chino], 技术与汉语教学刊物 [Journal of Technology and Chinese Language Teaching], vol. 1, núm. 1, 2010, pp. 71-93.

9 Todos ellos mencionados en Liu, "Second Life", op. cit., pp. 77, 78 y 79. Respectivamente: Scott Grant, “About Chinese Island”, Monash Chinese Island web, noviembre de 2010; Sachiko Kamioka, "MyChina: Summer Chinese Immersion Camp in Second Life", trabajo presentado en la 2009 ACTFL Annual Convention and World Languages Expo, 20-22 de noviembre, San Diego, California, 2009; Zheng Xiuren, Zhan Hong y Chen Qingxuan, “华语师资培训之教学个案研究一以虚拟实 境Second Life为例” [Estudio de casos de enseñanza al profesorado de chino a través de la realidad virtual de Second Life], 中原华语文学报 [Revista Literaria China de la Llanura Central], vol. 5, pp. 157-178. 
de Ning Zhao ${ }^{10}$ confirma la actualidad de esta relación. De este modo, el número de "islas" (espacios virtuales dedicados específicamente a un tema) culturales en Second Life llega a 63 y constituyen $3 \%$ de las existentes. De ellas, 52 se dedican a temas educativos y 11 a organismos no lucrativos. De éstas, $52 \%$ se enfocan a la enseñanza de conocimientos básicos, $21 \%$ a cursos específicos para estudiantes y $13 \%$ a la investigación en enseñanza y aprendizaje en ambientes virtuales. ${ }^{11}$ En cuanto a la enseñanza de CSL, la Universidad Estatal de Michigan estableció el Instituto Confucio Virtual en Second Life y la Universidad de Monash compró una isla virtual en 2007. Su Departamento de Estudios Chinos estableció una isla china que se dedica, específicamente, a la enseñanza y el aprendizaje de la lengua y la cultura chinas y se encuentra activa desde entonces. ${ }^{12}$

\section{Discusión y conclusiones}

En lo concerniente a las aplicaciones diseñadas para la enseñanza de CSL, destaca la tendencia a la colaboración de China y otros países para crear productos diversos y mejorar sus contenidos. El factor complementario en cuanto al refuerzo fonético y de vocabulario que estos sistemas ofrecen respecto al aprendizaje del aula es sobresaliente. En él descansa su principal fortaleza. El futuro, sin duda, se caracterizará por el incremento de estas herramientas entre los alumnos. Sin embargo, el proceso no podrá agilizarse si no se tienen en cuenta las principales deficiencias o debilidades: hay discrepancias gramaticales en diferentes aplicaciones que deberían estandarizarse; el contenido no presenta aún la mejor sistematicidad y no resultan sencillas en muchos casos; en las correspondientes a la prueba de HSK de suficiencia faltan prácticas $y$, sobre todo, se requieren aplicaciones profesionales de gramática china.

${ }^{10}$ Ning Zhao, “虚拟现实在对外汉语教学中的应用一以“第二人生”为例” [Aplicación de la realidad virtual a la enseñanza del chino como L2. Un estudio de caso de Second Life], tesis de maestría, Universidad de Shandong, 2015.

${ }^{11}$ Ibid., p. 20.

${ }^{12}$ Grant, "About Chinese Island”, op. cit. 
Desde el reconocimiento innegable de los beneficios que la realidad virtual aporta al trabajo tradicional de las aulas, se concluye que, de todos los programas mencionados $-\mathrm{y}$ a pesar de que las plataformas de realidad virtual han proliferado-, Second Life destaca por la acogida que ha tenido entre docentes y especialistas, quienes lo consideran el simulador más apropiado en la actualidad. Básicamente, sus potencialidades giran en torno al interés que genera en los aprendices y a los intercambios que fomenta entre ellos (eminentes fortalezas). De este modo, el sistema resulta muy eficaz y abierto, pues no hay restricción horaria para su uso. Sin duda, la principal crítica (entre sus debilidades) radica en la necesidad de aumentar el número de entornos desde donde potenciar la enseñanza de CSL. Un mayor número de "islas" es imprescindible. Además, entre sus desventajas, se subraya la conveniencia de acortar el periodo de adaptación al sistema, resolver los problemas y simplificar los requerimientos técnicos para acceder a la plataforma. De igual manera, se aconseja el incremento de profesores asistentes para apoyar a los docentes que diseñan actividades en red.

\section{Bibliografía}

CHEN, Liyu y Tan Yongyu, “数位资源辅助华语语音教学分析一以 香港粤语母语者为教学对象” [Análisis de recursos digitales de enseñanza y aprendizaje de la fonética en chino mandarín para los hablantes de chino cantonés], 第十二屆台灣華語文教學 年會暨國際學術研討會 [Acta del XII Congreso Internacional de Enseñanza y Aprendizaje de Chino de Taiwan], 屆台: 文藻外語 學院應用華語文系暨華語文教學研究所 [Taiwan, Facultad de Lenguas Extranjeras Aplicadas Wenzao, Instituto Superior de Enseñanza de Lengua China], 2013, pp. 351-361.

Grant, Scott, "About Chinese Island”, Monash Chinese Island web, noviembre de 2010. [ < http://www.virtualhanyu.com/?page_ $\mathrm{id}=87>$, consultado el 28 de marzo de 2016.]

Kamioka, Sachiko, "MyChina: Summer Chinese Immersion Camp in Second Life", trabajo presentado en la 2009 ACTFL Annual Convention and World Languages Expo, 20-22 de noviembre, San Diego, California, 2009.

LIANG, Zhizhi, “面向初级汉语水平外国学生词汇学习的手机汉语 
学习软件设计” [Diseño de software de teléfono inteligente para el aprendizaje de vocabulario chino para estudiantes extranjeros de nivel inicial], 广西教育学院学报 [Revista del Colegio de Educación de Guangxi], vol. 3, 2014, pp.152-155.

LiU, Shijuan, “Second Life 及其在中文教学中的应用” [Second Life y su aplicación en la enseñanza y en el aprendizaje del chino], 技 术与汉语教学刊物 [Journal of Technology and Chinese Language Teaching], vol. 1, núm. 1, 2010, pp. 71-93.

Moon, Youmi, “基于智能手机app的汉语口语智能学习模式设计 研究一以韩国初级汉语学习者为对象” [Estudio del uso de las aplicaciones para el aprendizaje inteligente: el caso de los estudiantes coreanos y su aprendizaje aplicado a conversación], 中文 教学现代化学报 [Revista para la Modernización de la Enseñanza de la Lengua China], vol. 4, núm. 2, 2015.

XIAO, Meng Gang, “汉语学习类手机应用使用情况调查研究一以孟 加拉达卡大学汉语学习者为例” [Investigación del uso de aplicaciones de teléfono inteligente de enseñanza de chino. Análisis basado en los alumnos de chino de la Universidad de Dhaka de Bangladesh], tesis de maestría, Universidad de Yunnan, 2015.

XIONG, Qianqian, “近十年来现代教育技术在汉语国际教育中的应 用及趋势” [Aplicación y tendencia de las tecnologías modernas en la enseñanza internacional de chino en la última década], tesis de maestría, Universidad de Estudios Extranjeros de Guangdong, 2014.

Zhang, Yi et al., "Educational Game Design for Teaching Chinese as a Foreign Language by Effective Learning Environment, Flow, Motivation", en Xiangfeng Luo et al. (eds.), New Horizons in Web-based Learning-ICWL 2010 Workshops, Berlín-Heidelberg, Springer, 2011.

ZHAO, Ning, “虚拟现实在对外汉语教学中的应用一以“第二人生”为 例” [Aplicación de la realidad virtual en la enseñanza del chino como L2. Un estudio de caso de Second Life], tesis de maestría, Universidad de Shandong, 2015.

ZHENG XIUREN, Zhan Hong y Chen Qingxuan, “华语师资培训之教 学个案研究一以虚拟实境Second Life为例” [Estudio de casos de enseñanza al profesorado de chino a través de la realidad virtual de Second Life], 中原华语文学报 [Revista Literaria China de la Llanura Central], vol. 5, pp. 157-178. 
